\title{
Estudo Comparativo de Dose de Radiação Aplicada a Pacientes nos Sistemas Convencionais e Sistemas Digitais de Imagem
}

\section{Comparative Radiation Dose Study Applied to Patients Conventional Systems and Digital Image}

Systems

Lillian Lettiere Bezerra Lemos Marques $^{1}$, Alyson Marcos Gelsleichter ${ }^{2}$, Joyce Nedochetko ${ }^{3}$, Luciana Machado Sebastião ${ }^{4}$, Janie Ourique Feijó ${ }^{5}$, Alexandre D’Agostini Zottis ${ }^{6}$, Rita de Cássia Flôr ${ }^{7}$

\section{Resumo}

A introdução dos sistemas digitais de imagem representa um grande avanço na área da imaginologia, por possuir diversas vantagens sobre o sistema convencional. No entanto, sua ampla faixa dinâmica, ao mesmo tempo que pode ser benéfica ao diminuir o número de repetição de exames, permite a superexposição sem deterioração da imagem, o que pode implicar em elevação de dose nos pacientes. Objetivou-se avaliar a exposição em diferentes sistemas de imagem, possibilitando aos profissionais das técnicas radiológicas refletir sobre a utilização de doses desnecessárias. Assim, realizou-se uma pesquisa qualitativa exploratória do tipo revisão integrativa, por meio de artigos indexados na Biblioteca Virtual em Saúde, e publicados de 2005 a

\footnotetext{
1 1.Tecnóloga em Radiologia; Mestre em Proteção Radiológica- Instituto Federal de Santa Catarina-IFSC; Especialização em Tomografia Computadorizada e Ressonância Magnética; Especialização em Docência, Gestão e Supervisão Escolar;lillianlbl@ gmail.com (86) 98101-6959

2.Tecnólogo em Radiologia; Mestre em Proteção Radiológica-Instituto Federal de Santa Catarina-IFSC; alyson.marcos@ gmail.com (48) 9919-9484

3.Tecnóloga em Radiologia; Mestre em Proteção Radiológica-Instituto Federal de Santa Catarina-IFSC; jnedochetko@gmail.com

4.Tecnóloga em Radiologia; Mestre em Proteção Radiológica-Instituto Federal de Santa Catarina-IFSC; lucianabr_2002@yahoo.com
}

2016, nos idiomas português e inglês. Adotouse como critério de inclusão a relação do material com a pergunta norteadora. Da execução da metodologia proposta, resultou a seleção de dez artigos científicos. Constatouse a unanimidade entre os autores de que há um aumento significativo da dose aplicada nos sistemas digitais de imagem em relação ao sistema convencional, devido, principalmente, à ampla faixa dinâmica desses sistemas. Concluiu-se pela necessidade de treinamento dos profissionais para utilização da dose correta e da adoção pelos serviços de imaginologia de gestão da dose, conforme recomendado pela Comissão Internacional de Proteção Radiológica. Descritores: Radiologia. Radiografia Digital. Dose. Proteção Radiológica. Gestão da qualidade.

5. Tecnóloga em Radiologia ; Discente do Programa de Pós GraduaçãoMestrado Profissional em Proteção Radiológica -MPPR, Departamento Acadêmico de Saúde e Serviço - DASS- IFSC-janiefeijo@gmail.com 6. Físico; Doutorado em Química Inorgânica da Universidade Federal de Santa Catarina; Docente do Instituto Federal de Santa Catarina-IFSC-adz@ifsc.edu.br 7. Enfermeira; Doutorado em Enfermagem; Docente do Instituto Federal de Santa Catarina-IFSC- flor@ifsc.edu.br. 
Revista Eletrônica Gestão \& Saúde ISSN: 1982-4785

\section{Abstract}

The introduction of digital imaging systems represents a major advance in the field of imaging, having several advantages over the conventional system. However, its wide dynamic range can favor the decrease in the number of repetitions, as it also allows for overexposure without image deterioration, which can lead to a tendency to increase the dose in patients. The objective of this study was to evaluate the exposure in different imaging systems, allowing radiological technicians to reflect on the use of unnecessary doses. Thus, a qualitative exploratory research characterized as integrative review was carried out by means of articles indexed in the virtual health library and published within 2005 and 2016 in the Portuguese and English languages. The article connection to the guiding question was adopted as inclusion criterion. After the accomplishment of the proposed methodology, ten scientific articles were selected. Authors agree unanimously on the significant increase in the dose applied in digital imaging systems in relation to the conventional system, mainly due to the wide dynamic range of those systems. We conclude for also the need to train professionals in the correct dose as the adoption of dose management by imaging services, as recommended by the international commission on radiological protection. Keywords: Radiology. Digital Radiography.
Estudo Comparativo de Dose de Radiação...

Dose. Radiological Protection. Quality management.

\section{Introdução}

A introdução dos Sistemas Digitais de Imagem, quando comparados à radiologia convencional, - o sistema Tela-Filme (TF) -, representa um avanço significativo na área da imaginologia, pois possuem vantagens como o armazenamento eletrônico, ampla faixa dinâmica e pós processamento da imagem ${ }^{(1-2)}$. Entretanto, algumas delas têm seu viés negativo, pois podem mascarar erros de aquisição da imagem, como superexposição e colimação inadequada ${ }^{(1,3,4)}$.

A faixa dinâmica pode ser definida como a proporção mínima e máxima de exposição que um dispositivo de imagem pode receber sem deteriorar ou distorcer a imagem. Como o sistema TF possui uma faixa dinâmica pequena, não permite que haja grandes variações de dose, sob pena de perda de qualidade da imagem. Já nos sistemas digitais de imagem a ampla faixa dinâmica permite grandes variações sem que se produza um efeito negativo na imagem. Contudo, subexposições aumentam os ruídos na imagem, enquanto as superexposições podem provocar melhoria na qualidade da imagem, favorecendo a relação entre sinal-ruído ${ }^{(3 ; 5)}$.

Ao mesmo tempo que a ampla faixa dinâmica dos sistemas digitais de imagem pode ser benéfica ao evitar repetições de 
Revista Eletrônica Gestão \& Saúde ISSN: 1982-4785 Marques LLB, Gelsleichter MA, Nedochetko J et al

exames, pode gerar uma tendência de elevação de doses nos pacientes por permitir exposição excessiva sem afetar a qualidade da imagem (3,6). Imagens com superexposição ou colimação pequena podem ser corrigidas com o tratamento digital, enquanto as imagens subexpostas ou com colimação insuficiente demandarão repetições. Dessa forma, os Profissionais das Técnicas Radiológicas tendem a abusar dos fatores de exposição e da $\operatorname{colimação~}^{(3 ; 4)}$.

O desenvolvimento de métodos práticos para a avaliação de doses em pacientes é de extrema importância e faz parte dos Programas de Controle de Garantia em Qualidade (PCGQ) obrigatoriamente implantados em toda instituição médica que faça uso de radiações ionizantes. No Brasil, a Portaria $n^{\circ}$ 453/98 SVS/MS é o instrumento legal para esse fim ${ }^{(3 ; 5 ; 7)}$.

Apesar do progresso da tecnológico do campo da radiologia, com a introdução e popularização de exames complexos como a tomografia computadorizada e ressonância magnética, as radiografias simples ainda representam até $80 \%$ de todos os estudos. A radiologia digital tem maior custo em relação à radiologia convencional. Este investimento é justificado se todas as seguintes vantagens da tecnologia digital forem levadas em consideração e incorporadas à rotina diária: qualidade de imagem superior e menor dose no paciente; fluxo de trabalho mais eficiente,
Estudo Comparativo de Dose de Radiação...

teleradiologia; redução do espaço de armazenamento de imagens e estudos que durem menor tempo e propiciem mais conforto ao paciente $^{(3)}$. Embora a radiologia digital tenha o potencial de reduzir a dose no paciente a experiência mundial tem demonstrado que as doses praticadas não diminuíram e sim aumentaram, pois, até certo nível, a superexposição em sistemas digitais de imagem pode apresentar boa qualidade imagem $^{(3)}$.

Assim, o objetivo deste trabalho é revisar a literatura em busca de estudos que avaliem a dose utilizada em diferentes sistemas de imagem, a fim de reunir dados suficientes para reflexão dos profissionais das técnicas radiológicas com relação à utilização de níveis de dose desnecessários, ou seja, exposições que não agregam benefícios ao propósito clínico esperado.

\section{Revisão de Literatura}

As imagens radiológicas são extremamente importantes para aplicar um diagnóstico correto através das alterações e patogenias existentes. Para isso, é necessário obter um bom nível de qualidade da imagem e seguir os três princípios recomendados pela Comissão Internacional em Proteção Radiológica (International Commissionon Radiological Protection- ICRP), que são: a justificação, a otimização e a aplicação de limites de dose ${ }^{(8 ; 9)}$. 
Revista Eletrônica Gestão \& Saúde ISSN: 1982-4785

Marques LLB, Gelsleichter MA, Nedochetko J et al

Os dois primeiros princípios são aplicados a qualquer situação de exposição à radiação e afirmam, respectivamente, que um procedimento deverá ser realizado apenas se os benefícios da exposição médica do paciente forem maiores que os possíveis malefícios e que a dose aplicada deve ser a mínima possível para uma qualidade de imagem aceitável. O terceiro princípio é aplicado, além do público, à equipe de profissionais sujeita a exposições ocupacionais, pois sofre diversas exposições ao longo da carreira, sendo necessário não exceder os limites estabelecidos, de forma a se manter um nível de proteção apropriado ${ }^{(9)}$.

A qualidade da imagem está ligada a uma série de fatores físicos e operacionais que, quando bem combinados, acarretam em diagnóstico preciso de patogênese e níveis seguros de operação para todos os envolvidos no procedimento, tendo em vista que a radiação é um ente invisível e inodoro além de ser acumulativo e ter potencial destrutivo e mutagênico no organismo. Sabendo-se disso, com o passar dos anos, surgiu a preocupação com as doses de radiação pela classe médica e científica de forma que, se passou a perseguir a boa imagem com a menor dose possível além da otimização dos equipamentos bem e da implantação de normas pertinentes ao uso adequado da radiação e até das novas técnicas empregando o mesmo princípio, como por exemplo, a Radiografia Digital ${ }^{(10)}$.
Estudo Comparativo de Dose de Radiação...

\section{Formação da imagem nos sistemas de Raios X}

\section{Radiologia convencional}

A imagem radiográfica no sistema convencional segue uma sequência de procedimentos. A primeira etapa consiste na emissão do feixe de radiação pelo foco emissor até o objeto ou paciente. Esse feixe de radiação interage com o objeto, onde ocorre a atenuação do feixe de raios $X$. Na terceira etapa o feixe de radiação emerge do objeto, sensibilizando os cristais de prata do filme que está posicionado posteriormente à estrutura. $\mathrm{O}$ filme sensibilizado é então submetido à revelação química que composta pelas seguintes etapas: Revelação com solução básica; Fixação com solução ácida, Lavagem com água para remover o excesso de químicos e, enfim, a Secagem. ${ }^{(8,11.12)}$.

\section{Radiologia Computadorizada (CR)}

O Sistema CR utiliza cassetes eletrônicos semelhantes ao sistema convencional no qual o conjunto de filmeécran é substituído por um sistema de fósforos com características de luminescência fotoestimulável. A placa de fósforo armazena a energia dos fótons de raios $\mathrm{X}$ e as libera em forma de luz após receber o estímulo de uma 
Revista Eletrônica Gestão \& Saúde ISSN: 1982-4785

luz de infravermelho. Esse modo de revelar a imagem ocorre de modo automático.

Esse sistema utiliza cassetes com placas de imagens reutilizáveis, eliminando o custo com os filmes radiográficos. As imagens obtidas são digitalizadas e exibidas no monitor de uma estação de trabalho, podem ser manuseadas, transferidas ou armazenadas ${ }^{(13)}$.

\section{Radiologia Digital Direta (DR)}

A imagem radiográfica digital é obtida pela conversão do feixe de radiação em sinais elétricos. Esse processo é realizado por meio de detectores acoplados ao aparelho de raios $\mathrm{X}$ e não requer uso de chassis ou cassetes, pois utiliza-se de uma tecnologia de leitura direta e instantânea na aquisição da imagem através de detectores CCD (Charge-Coupled Device) ou de detectores digitais no painel plano ${ }^{(11)}$.

Os sistemas digitais empregam método não poluente e ecologicamente correto, dado que não necessitam de processamentos químicos na revelação na imagem As imagens são enviadas diretamente para o computador em forma de sinais elétricos e visualizadas quase que instantaneamente no monitor com uma melhor resolução e qualidade, fazendo com que a produtividade seja superior ao sistema radiográfico convencional, já que economiza tempo de transporte e processamento das imagens. Outro beneficio que o paciente dispõe nesse sistema, é a
Estudo Comparativo de Dose de Radiação...

possibilidade de obter um laudo mais rápido pelo especialista, pois a imagem pode ser transmitida de um local a outro ${ }^{(8,11)}$.

\section{Metodologia}

Este estudo adotou pesquisa qualitativa exploratória do tipo revisão integrativa, método difere da revisão tradicional, especialmente pelo seu rigor científico, uma vez que, durante todas as etapas da pesquisa, tenta superar possíveis vieses. Trata-se de um instrumento utilizado na prática baseada em evidências que possibilita a construção do conhecimento científico sobre determinado assunto que não está suficientemente fundamentado ${ }^{(14 ; 15)}$.

Durante sua elaboração, é necessária a adoção de fases que fortaleçam o rigor metodológico da busca, devendo seguir seis passos operacionais: (i) identificação do problema ou questionamento com a elaboração da pergunta norteadora; (ii)estabelecimento de critérios de inclusão e exclusão de artigos por meio da busca ou amostragem na literatura; (iii) coleta de dados com a definição das informações a serem extraídas dos artigos selecionados; (iv) análise crítica das informações dos estudos incluídos; (v) interpretação e discussão dos resultados e; a última etapa consiste na (vi) apresentação da revisão integrativa ${ }^{(15)}$.

O questionamento norteador que guiou a construção deste estudo foi se "A introdução 
Revista Eletrônica Gestão \& Saúde ISSN: 1982-4785

dos sistemas digitais de imagem alteraram o nível de dose nos pacientes" A fase de seleção dos artigos se iniciou no mês de abril de 2016 na Biblioteca Virtual em Saúde, utilizando-se os descritores padronizados pelo DeCS (Descritores em Ciências da Saúde): Radiologia, Proteção Radiológica, Radiobiologia, bem como seus correspondentes no idioma inglês, com o auxílio da expressão booleana: 'AND' para a inserção de duas ou mais palavras. Após a aplicação dos descritores, nesse momento sem nenhum critério de inclusão ou exclusão, foram selecionados 63 artigos. Entretanto, foram definidos alguns critérios de inclusão para facilitar e captar apenas os artigos relacionados ao objetivo da revisão integrativa, quais sejam, publicados em português e inglês, material disponível na íntegra para posterior análise, relação com a pergunta norteadora e recorte temporal dos anos de 2005 a 2016. Dessa forma, dez artigos compuseram a amostra final deste estudo.

\section{Resultados}

Pesquisadores compararam as exposições de duas incidências radiológicas tórax e coluna lombar - usando três sistemas de imagem - TF, CR e DR. Resultados demonstraram que as doses para as incidências de coluna lombar foram maiores no sistema CR do que nos sistemas DR e TF. Nas
Estudo Comparativo de Dose de Radiação...

incidências de tórax, a dose para TF foi maior quando comparada com outros sistemas ${ }^{(6)}$.

Outro estudo realizado em hospital no Estado do Rio Grande do Sul comparou a dose de entrada na pele nos sistemas TF e CR para várias incidências radiográficas. Primeiramente foi estabelecido, com base nas opiniões de um físico do serviço e dos médicos radiologistas, um nível de qualidade diagnóstica para as imagens digitais. $\mathrm{O}$ fator mais importante para estabelecer esse padrão foi o nível de ruído. Estabelecido o padrão, os fatores de exposição foram reproduzidos para aquela incidência e espessura de paciente. Essa técnica pôde então ser comparada com a anteriormente utilizada no sistema $\mathrm{TF}^{(16)}$. Os resultados comprovaram um aumento de dose com o sistema CR que variou de $27,55 \%$ para incidência de tórax em perfil, em um dos equipamentos de Raios X, a até 583,63\% para incidência Waters de seios da face, em outro equipamento de Raios X de alta frequência ${ }^{(16)}$.

No estudo comparativo das técnicas de exposição e doses entre um hospital brasileiro e três australianos, foram encontrados aumentos de dose de até 43 vezes no hospital brasileiro. Segundo os autores, uma simples mudança nos fatores de exposição empregados poderia contribuir significativamente para a redução dessas doses ${ }^{(8)}$.

Ainda segundo esse estudo, a dose no Brasil não é considerada fator limitante, sendo o principal fator a qualidade de imagem, 
Revista Eletrônica Gestão \& Saúde ISSN: 1982-4785

portanto, altos valores de mAs são utilizados para obtenção de um bom contraste. O estudo também concluiu que existe grande potencial de redução de dose, pois apesar de os hospitais australianos possuírem modernos equipamentos digitais, não obtiveram as menores doses nos exames de tórax ${ }^{(8)}$

\section{Discussão}

Apesar do potencial que os sistemas digitais de imagem apresentam por possibilitar uma redução de exposição no paciente, vários estudos evidenciam aumento de dose nessa modalidade de radiodiagnóstico e tal fato deve-se, principalmente, a ampla faixa dinâmica dos sistemas digitais em relação ao

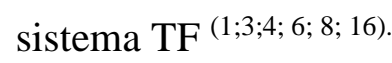

Nos sistemas digitais de imagem o aumento da faixa dinâmica torna mais difícil identificar uma sub ou superexposição. Portanto a instalação desses sistemas demanda atenção por parte dos serviços de radiodiagnóstico e treinamento adequado da equipe de profissionais da técnicas radiológicas. No caso de equipamentos com controle automático de exposição, devido à sensibilidade diferente à energia dos raios $\mathrm{x}$ dos sistemas digitais de imagem em relação ao sistema TF o controle de exposição automático deveria ser reprogramado. Em função disso, é
Estudo Comparativo de Dose de Radiação...

importante possuir protocolos de prova de controle de exposição automático de exposição, incluídos fantomas, e informações sobre o desempenho do gerador de raios-X com os detectores digitais e programas de garantia da qualidade ${ }^{(3)}$.

A ampla faixa dinâmica dos sistemas digitais de imagem permite uma vasta gama de doses no paciente e no detector, sem efeitos adversos na imagem. Isso não se aplica ao sistema $\mathrm{TF}$, onde o resultado de aumento na dosagem implica a obtenção de imagem com alta densidade radiográfica. Por outro lado, redução substancial na exposição resulta em imagem com baixa densidade. Ambas as situações podem inviabilizar a imagem para o diagnóstico. O sistema CR tem uma resposta linear para exposição à radiação de quatro ordens de grandeza em relação ao sistema TF (3).

No sistema TF a resposta do filme à exposição é representada pela curva sensitométrica, cuja faixa dinâmica é definida como o intervalo linear da curva. Os detectores digitais apresentam resposta mais ampla e linear à exposição (Figura 1), ou seja, permitem variação muito maior de dose, melhorando sua função com o aumento da $\operatorname{exposição~}^{(5)}$. 
Figura 1: Comparação entre a faixa dinâmica dos sistemas TF e dos detectores digitais ${ }^{(5)}$.

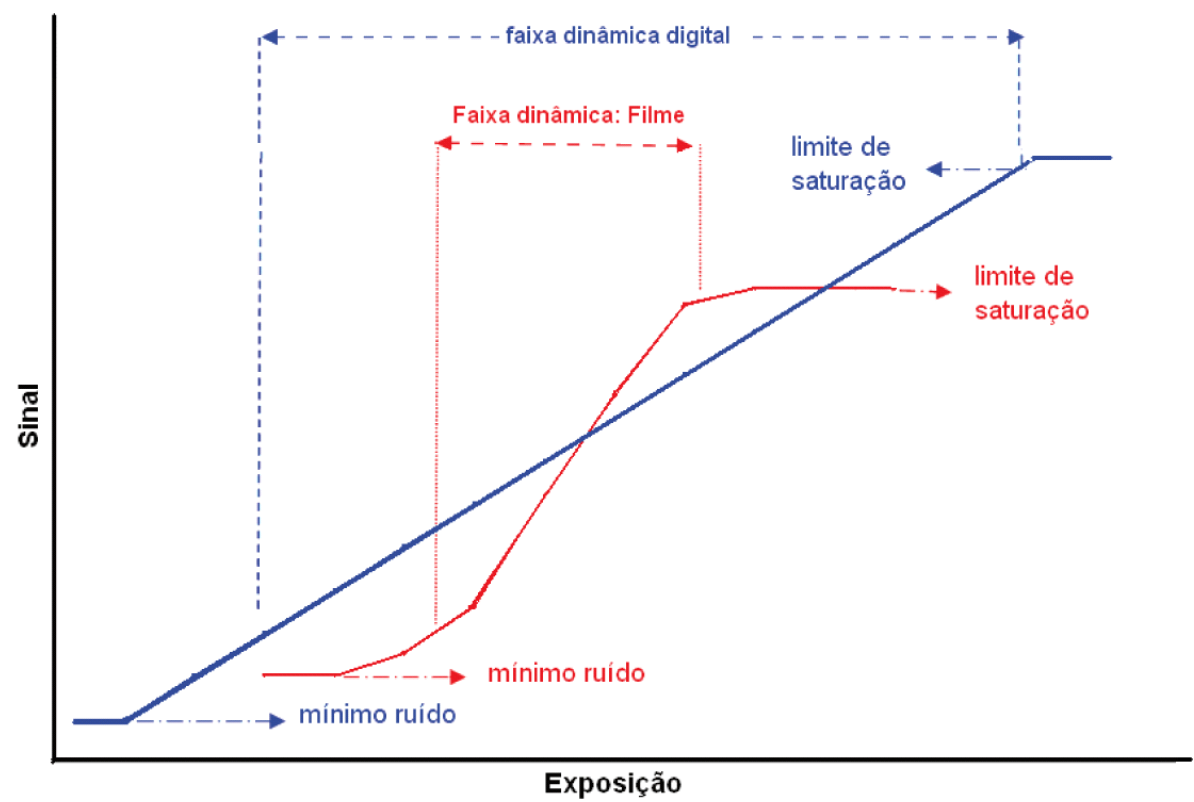

Observa-se também que nos sistemas digitais é utilizada uma maior faixa de carga de trabalho (mAs) do que no sistema convencional. Isso se deve ao fato que, no sistema TF, um aumento na intensidade da radiação acarreta a saturação da densidade óptica do filme e não haverá imagem com contraste adequado para o diagnóstico. No caso dos sistemas digitais, a faixa dinâmica dos detectores digitais é ampla, resultando sempre em imagem com contraste adequado independentemente de a intensidade ser alta ou baixa. Além disso quanto maior a intensidade da radiação melhor é a relação sinal-ruído ${ }^{(17)}$.

À vista disso, a conduta passiva dos profissionais de não explorarem o potencial de otimização da dose que possuem os detectores digitais, os quais permitem redução de dose sem afetar significativamente a qualidade de imagem, comparado ao sistema $\mathrm{TF}$, resulta em doses superiores ao sistema convencional ${ }^{(18)}$.

Os resultados dos estudos apresentam alta relevância ao se associar o aumento de dose ao consequente aumento da probabilidade de ocorrência de efeitos estocásticos. É provável que as exposições médicas produzam aumento muito pequeno de incidência de câncer na população, tornando-se plausível desatentar para os possíveis danos biológicos advindos de doses excessivas que objetivem a melhor qualidade de imagem em sistema digital $^{(14)}$. Tal escolha contraria visivelmente o princípio ALARA (As Low As Reasonably Achievably), que afirma que as doses empregadas devem ser as mínimas para a obtenção de diagnóstico ${ }^{(19)}$.

\section{Conclusão}


A partir da análise das pesquisas sobre doses usadas em pacientes nos sistemas TF, CR e DR verificou-se que a maioria dos artigos concorda que há significativo aumento da dose nos sistemas digitais de imagem em relação aos convencionais. Verificou-se também que mais do que as próprias características dos equipamentos, as condições de trabalho e a atitude dos profissionais das técnicas radiológicas, podem influenciar a proteção radiológica.

Nesse sentido, temos a necessidade de treinamento contínuo dos profissionais, para que pratiquem o princípio ALARA nessas novas tecnologias, alcançando, por consequência, a redução de danos biológicos.

Outro fator que pode impactar positivamente a proteção radiológica, principalmente com a adoção dos sistemas digitais de imagem, seria a adoção por parte dos serviços de programas de gestão da qualidade e gestão de doses conforme recomendado pela ICRP 93. Contudo, esperase que a leitura deste trabalho possa contribuir para o conhecimento científico no que tange à relevância da otimização de dose nos sistemas digitais de imagem, bem como para compreensão cada vez maior da importância da otimização da proteção radiológica em favor da melhoria da saúde da população em geral. 
Revista Eletrônica Gestão \& Saúde ISSN: 1982-4785

Marques LLB, Gelsleichter MA, Nedochetko J et al
Estudo Comparativo de Dose de Radiação...

\section{Referências}

1. Luz RM, Hoff G. Estudo comparativo da qualidade da imagem e do kerma, de entrada e de saída, em simulador de tórax utilizando sistemas analógico e digitalizado CR de aquisição de imagens. Radiol Bras. 2010;43:39-45.

2. Lee SC, Wang JN, Liu SC, Jiang SH. Evaluation of dose-image-quality optimization in digital chest radiography. Nuclear Instruments and Methods in Physics Research Section A: Accelerators, Spectrometers, Detectors and Associated Equipment. 2007;580(1):544-7.

3. International Commission on Radiological Protection (ICRP). Managing patient dose in digital radiology: a report of the International Commission on Radiological Protection. ICRP Publication 93. Ottawa;2004.

4. Uffmann M, Schaefer-Prokop C. Digital radiography: The balance between image quality and required radiation dose. Eur $\mathrm{J}$ Radiol. 2009;72(2):202-8.

5. Furquim TAC, Costa PR. Garantia de qualidade em radiologia diagnóstica. Rev Bras Fis Med. 2009;3(1):91-9.

6. Mohamed-Ahmed E, Osman H, Sulieman A. Evaluation of patient doses in conventional, computed and digital radiography. In: International Congress of International Radiation Protection Association, n.13, 2012, Glasgow. Anais. Glasgow, 2012. Disponível em: <http://www.irpa.net/members/P07.131fp.p df>. Acesso em: 14 mai. 2016.

7. Benefield LE. Implementing evidencebased practice in home care. Home Healthcare Nurse. 2003;21(12):804-9.

8. De Albuquerque AS, Santos AMS, Camelo CMA, Silva GG, Magalhães TMS, Araújo VGP, et al. Estudo comparativo entre sistemas radiográficos convencionais e digitais; revisão de literatura. Caderno de Graduação-Ciências Biológicas e da SaúdeFACIPE.2017;2(3):99.

9. International Commission on Radiological Protection (ICRP)., Radiological.ICRP publication 103. Ann. ICRP. Madrid; 2007.
10. Candeiro GTM, Bringel ASF, Vale IS. Radiologia Digital: Revisão De Literatura. Revista Odontológica de Araçatuba. 2009;30(2):38-44.

11. Biasoli Junior A. Técnicas Radiográficas: Princípios Físicos, Anatomia Básica, Posicionamento, Radiologia Digital, Tomografia Computadorizada. Rio de Janeiro: Editora Rubio; 2015.

12. Bontrager KL, Lampignan JP. Tratado de Técnica Radiológica e Anatomia Associada.(tradução Alcir Costa Fernandes, Douglas Omena Futuro, Fabiana Pinzetta). $8^{\mathrm{a}}$ ed. Rio de janeiro: Elsevier; 2015.

13. Robinson A. Vantagens e desvantagens da radiografia computadorizada. Disponível em:

http://www.ehow.com.br/vantagensdesvant agens- radiografia-computadorizadalista_7841/. Acesso em: 21 de junho de 2016.

14. Mendes KDS, Silveira RCCP.; Galvão CM. Revisão integrativa: método de pesquisa para a incorporação de evidências na saúde e na enfermagem. Texto Contexto Enferm. 2008:17(4):758-64.

15. Schimidt GT, Paula V. Doses de exposição em exames radiológicos realizados em sistemas CR e tela-filme. Disc Scientia. 2011;12(1): 65-75.

16. Körner M, Weber $\mathrm{CH}$, Wirt S, Pfeifer KJ, Reiser MF, Treitl M. Advances in digital radiography: physical principles and system overview. Radiographics. 2007;27(3):67586.

17. De Azevedo ANP, Mohamadain KEM, Osibote OA, Cunha ALL, Pires Filho A. Estudo comparativo das técnicas radiográficas e doses entre o Brasil e a Austrália. Radiol Bras. 2005;38(5):343-6.

18. Osibote AO. Avaliação das doses de radiação em pacientes adultos e pediátricos em exames de radiodiagnóstico. [tese na internet]. Rio de Janeiro: Escola Nacional de Saúde Pública Sérgio Arouca, Fundação Oswaldo Cruz; 2006. Disponível em: <http://www.arca.fiocruz.br/xmlui/bitstrea $\mathrm{m} /$ handle/icict $/ 4366 / 269$.pdf? sequence $=2>$. Acesso em: 21 mai. 2016.

19. Brasil. Agência Nacional de Vigilância Sanitária. Portaria MS/SVS no 453 , de $1^{\circ}$ de 
Revista Eletrônica Gestão \& Saúde ISSN: 1982-4785 Marques LLB, Gelsleichter MA, Nedochetko J et al

junho de 1998. Brasília: Diário Oficial da União, 2 jun.1998. Disponível em: <http://www.conter.gov.br/uploads/legislati vo/portaria_453.pdf.> Acesso em: 28 maio 2017.

Recebido: 20.06 .2018

Revisado: 31.07 .2018

Aprovado: 20.08.2018
Estudo Comparativo de Dose de Radiação...

\section{Participação dos autores:}

MARQUES, LLB trabalhou na concepção teórica, coleta e análise dos dados, elaboração e redação final do texto.

GELSLEICHTER, MA trabalhou na concepção teórica, coleta e análise dos dados, elaboração e redação final do texto.

NEDOCHETKO J trabalhou na concepção teórica, coleta e análise dos dados, elaboração e redação final do texto.

SEBASTIÃO, LM trabalhou na concepção teórica, coleta e análise dos dados, elaboração e redação final do texto.

FEIJÓ, OJ trabalhou na concepção teórica, coleta e análise dos dados, elaboração e redação final do texto. FLÔR, RC trabalhou na concepção teórica e supervisionou como coorientadora a confecção do trabalho.

ZOTTIS, AD trabalhou na concepção teórica e supervisionou como orientador a confecção do trabalho. 